\title{
Medikamentös induzierte Psychosen
}

\author{
Christian Prüter
}

Krankenhaus Maria Hilf, Fachkrankenhaus

für Psychiatrie und Psychotherapie, Gangelt

psychoneuro 2005; 31 (9): 422-425

Es gibt eine Vielzahl von Medikamenten, die unerwünschte psychische Arzneimittelwirkungen (UAW) auslösen können. Diese Medikamente werden häufig in anderen Fachdisziplinen als der Psychiatrie eingesetzt und verordnet. Viele Medikamente können das zentrale Nervensystem beeinflussen und auch psychotische Zustandsbilder verursachen. Häufig werden solche UAWs übersehen, nicht als Medikamenteneffekt identifiziert oder fälschlicherweise als Ausdruck einer eigenständigen psychischen Störung missinterpretiert. Der folgende Artikel gibt eine Übersicht über die wichtigsten Medikamentengruppen, die eine psychotische Symptomatik induzieren können, und Hinweise zur Therapie.

$\mathrm{E}$ s gibt eine Vielzahl von Medikamenten, die unerwünschte psychische Arzneimittelwirkungen (UAW) auslösen können. Diese Medikamente werden häufig im Bereich der Inneren Medizin, Dermatologie und Anästhesie eingesetzt und verordnet (63). Medikamente, die psychotrop wirksam sind, können das zentrale Nervensystem beeinflussen und in seltenen Fällen auch psychotische Zustandsbilder verursachen. Häufig werden solche UAWs übersehen, nicht als Medikamenteneffekt identifiziert oder fälschlicherweise als Ausdruck einer eigenständigen psychischen Störung missinterpretiert.

In der psychiatrischen Terminologie wurden seit Bonhoeffer (5) alle psychiatrischen Syndrome, die in Folge einer körperlich angreifenden Noxe akut auftreten, als „akute exogene Reaktionstypen“ beschrieben. Bonhoeffer betonte die Noxenunspezifität der akuten exogenen Reaktionstypen sowie die Symptomunspezifität, d.h. unabhängig von der speziellen Ätiologie können die gleichen psychiatrischen Syndrome auftreten.

Für die Diagnose einer medikamenteninduzierten Psychose spricht insbesondere ein enger zeitlicher Zusammenhang mit der Medikamenteneinnahme und ein schnelles Abklingen der Psychose nach Absetzen des Medikaments $(6,28)$. Bislang gibt es keine größeren systematischen Studien zur Prävalenz und Inzidenz medikamenteninduzierter Psychosen. In der Fachliteratur sind aber zumeist Kasuistiken und einzelne Verläufe vorzufinden. Trotz der Einrichtung eines Spontanerfassungssystems für unerwünschte Arzneimittelwirkungen seitens der Deutschen Arzneimittelkommission muss aber eine hohe Dunkelziffer nicht dokumentierter Fälle angenommen werden.

Die medikamenteninduzierten Psychosen sind nach den Internationalen Klassifikationssystemen unter die Diagnose des Delirs einzuordnen. Nach den heute gültigen internationalen diagnostischen Klassifikationen bezeichnet die Diagnose Delir (Tab. 1) alle psychischen Störungen, die organische Ursachen haben und mit Störungen des Bewusstseins, der Aufmerksamkeit und der Kognition einhergehen. Insbesonseins ergibt sich neben dem akuten Auftreten der Symptome die diffedere in der Störung des Bewusst- renzialdiagnostische Abgrenzung zur Demenz, wobei delirante $\mathrm{Zu}$ standsbilder häufig komorbid mit einer Demenz auftreten (17). Im klinischen Alltag ist die sichere differenzialdiagnostische Zuordnung zwischen „endogener“ Psychose und „exogener“ psychischer UAW als Folge einer medikamentösen Behandlung gelegentlich schwierig.

Delirien stellen in Allgemeinkrankenhäusern vor allem in den chirurgischen und internistischen Abteilungen ein Problem da. Nach Untersuchungen (33) kann bei 10$30 \%$ der älteren Patienten, die in ein Allgemeinkrankenhaus aufgenommen werden, ein Delir diagnostiziert werden. Je nach Art des Eingriffes schwanken die Inzidenzraten für ein postoperatives Delir zwischen 20 bis $60 \%(15,19)$. Klinisch wird das Delir durch Störungen von Bewusstsein, Aufmerksamkeit, Kognition, Wahrnehmung, Psychomotorik, SchlafWach-Rhythmus und Affektivität gekennzeichnet. Ein Delir entwickelt sich zumeist plötzlich, z.B. einige Stunden nach Medikamenteneinnahme oder innerhalb von $48 \mathrm{~h}$ nach einer größeren Operation. Je langsamer die Noxe wirkt, um so verzögerter kommt es zum Auftreten der deliranten Symptomatik. Bei täglicher Einnahme eines niedrig dosierten anticholinerg wirksamen Medikaments tritt ein Delir auf, wenn es zur entsprechenden Überschreitung eines gewissen individuell variablen Gewebespiegels der jeweiligen Substanz kommt. Delirien im Anschluss an operative Eingriffe werden am häufigsten am zweiten post- 
operativen Tag beobachtet. Substanzentzugsdelirien treten unter Berücksichtigung der jeweiligen Eliminationshalbwertszeit meist einige Stunden bis Tage nach dem abrupten Absetzten (z.B. bei Tranquilizern) auf. Die Diagnose wird klinisch gestellt, es empfiehlt sich aufgrund der mannigfaltigen Ursachen eines Delirs neben der psychopathologischen und körperlich-neurologischen Untersuchung je nach Symptombild eine Reihe weiterer diagnostischer Maßnahmen durchzuführen (Tab. 2). Bei Patienten mit hoher Vulnerabilität kann ein Delir durch weit geringere oder weniger Noxen ausgelöst werden als bei einem Patienten mit geringer Vulnerabilität. Daher kommen Delirien auch bei jungen Menschen vor, insbesondere nach schwierigen und komplikationsreichen Operationen.

Besonders häufig werden Delirien durch eine Vielzahl von Medi- kamenten ausgelöst, die entweder das monoaminerge System stimulieren oder im cholinergen System hemmend wirken. Die resultierenden Krankheitsbilder werden als zentrale anticholinerge Syndrome (ZAS) bezeichnet. In verschiedenen Studien waren Medikamente in 1130\% der Patienten, die ein Delir entwickelten, Ursache der Symptomatik $(18,20,45)$. Insbesondere Polypharmazie war mit dem erhöhten Risiko eines Delirs verbunden. So wurde bei Verordnung von mehr als drei Medikamenten während des stationären Aufenthaltes ein relatives Risiko von 2,9 berichtet, ein Delir zu entwickeln (25).

\section{- Hypnotika und Sedativa}

Benzodiazepine können sowohl bei Entzug wie als Nebenwirkung der Therapie Delirien verursachen (38). Im Entzug entwickelt sich ein Delir je nach Halbwertszeit des ein- genommenen Präparates etwa zwei bis zehn Tage nach Absetzen und erreicht schnell ein Maximum. Ein solches Entzugsdelir kann fünf bis zehn Tage andauern und ist gekennzeichnet durch:

- Verwirrtheitszustände

- Depersonalisation/Derealisation

- psychotische Symptome (Halluzinationen)

- ängstlich-depressive Syndrome

- Krampfanfälle

- Oszillopsien, Dysmorphopsien

- Photophobie

- Hyperakusis

- Hypersomnien

- Dsyästhesien

- kinästhetische Störungen

- Muskelzittern und -faszikulationen.

Nach etwa viermonatiger Einnahme auch einer therapeutischen Dosis von Benzodiazepinen kann es bei abruptem Absetzen zu einer Ent-

\section{Tab. 1 Diagnostische Kriterien des Delirs nach ICD-10 und DSM-IV}

ICD-10

1. Bewusstseinsstörung, d.h. verminderte Klarheit der Umgebungswahrnehmung mit eingeschränkter Fähigkeit, die Aufmerksamkeit zu richten, aufrechtzuerhalten und zu verlagern.

2. Globale Störung der Kognition, Wahrnehmungsstörungen, wie Verzerrungen der Wahrnehmung, Illusionen und meist optischen Halluzinationen; Beeinträchtigungen des abstrakten Denkens und der Auffassung, mit oder ohne flüchtige Wahnideen, aber typischerweise mit einem gewissen Grad an Inkohärenz; Beeinträchtigung des Arbeits- und Kurzzeitgedächtnisses, mit relativ intaktem Langzeitgedächtnis; zeitlicher Desorientiertheit, in schweren Fällen auch Desorientierung zu Ort und Person.

3. Psychomotorische Störungen (Hypo- oder Hyperaktivität mit nicht vorhersehbarem Wechsel zwischen beiden; verlängerte Reaktionszeit; vermehrter oder verminderter Redefluss; verstärkte Schreckreaktion.

4. Störungen des Schlaf-Wach-Rhythmus (Schlafstörung, in schweren Fällen völlige Schlaflosigkeit, mit oder ohne Schläfrigkeit am Tage oder Umkehr des Schlaf-Wach-Rhythmus, nächtliche Verschlimmerung der Symptomatik, unangenehme Träume oder Albträume, die nach dem Erwachen als Halluzinationen oder Illusionen fortbestehen können).

5. Affektive Störungen wie Depression, Angst oder Furcht, Reizbarkeit oder Euphorie, Apathie oder staunende Ratlosigkeit.

6. Das Störungsbild entwickelt sich innerhalb einer kurzen Zeitspanne (gewöhnlich innerhalb von Tagen oder Stunden) und fluktuiert üblicherweise im Tagesverlauf, die Gesamtdauer der Störung beträgt weniger als sechs Monate.

7. Eine ziemlich zuverlässige Diagnose des Delirs kann sogar dann gestellt werden, wenn die zugrunde liegende Ursache nicht nachzuweisen ist.

\section{DSM-IV}

A. Bewusstseinsstörung, d.h. verminderte Klarheit der Umgebungswahrnehmung mit eingeschränkter Fähigkeit, die Aufmerksamkeit zu richten, aufrechtzuerhalten und zu verlagern.

B. Veränderungen der kognitiven Funktionen (wie Gedächtnisstörung, Desorientiertheit oder Sprachstörung) oder Entwicklung von Wahrnehmungsstörungen (Illusionen, Halluzinationen, darauf aufbauenden Wahnideen), die nicht besser durch eine schon vorher bestehende Demenz erklärt werden können.

C. Das Störungsbild entwickelt sich innerhalb einer kurzen Zeitspanne (gewöhnlich innerhalb von Tagen oder Stunden) und fluktuiert üblicherweise im Tagesverlauf.

D. Es gibt Hinweise aus der Anamnese, der körperlichen Untersuchung oder den Laborbefunden, dass das Störungsbild direkte körperliche Folgeerscheinung eines medizinischen Krankheitsfaktors ist. 
zugssymptomatik kommen, die allerdings nicht immer so ausgeprägt sein muss wie oben geschildert (Inzidenz $<5 \%$ ). Insbesondere das abrupte Absetzen kurzwirksamer Benzodiazepine nach Aufnahme in ein Krankenhaus ist eine häufige Ursache für ein Delir $(18,45)$.

\section{Antikonvulsiva}

Psychotische Syndrome und Verwirrtheitszustände treten hauptsächlich unter Phenytoin, Primidon und Phenobarbital auf (58), bei Überdosierungen und Intoxikationen auch unter Valproat und Carbamazepin (22). Die neueren Antikonvulsiva scheinen insgesamt eine geringere delirogene Potenz zu haben, dennoch liegen sowohl für Lamotrigin (40) als auch für Topiramat (37, 41) kasuistische Berichte über psychotische Syndrome vor.

\section{Antiparkinsonmedikamente}

Verwirrtheitszustände, delirante Syndrome und Psychosen sind häufige Komplikationen der medikamentösen Therapie vor allem des fortgeschrittenen Morbus Parkinson (68). Ätiologisch ist neben der medikamentösen Therapie auch die Erkrankung selbst oder eine komorbide Demenz zu berücksichtigen. Als Risikofaktoren für ein Delir bei Parkinsonpatienten gelten fortgeschrittenes Alter, eine Demenz und eine Kombinationsbehandlung mit verschiedenen Antiparkinsonmedikamenten sowie eine hohe Dosierung dieser Präparate. Alle in der Parkinsontherapie eingesetzten Medikamente können Delirien und dopaminerge Psychosen auslösen. Bei etwa 5\% der Patienten, die mit Standarddosierungen von Levodopa behandelt werden, entwickelt sich ein Delir $(7,10)$. Für den Dopaminagonisten Pergolid werden Inzidenzraten von $11-33 \%(34,31)$ und für Bromocriptin von $12 \%$ (35) für delirante Syndrome berichtet. Auch unter Therapie mit Amantadin und Selegilin treten häufiger Delirien auf (10). In einer weiteren Studie (26) kam es zum Auftreten deliranter Symptomatik während der Umstellung von unretardierten auf retadierte L-Dopapräparate. Vom Delir sind die im Spätverlauf der Erkrankung bei 20-

\section{Tab. 2 Untersuchungen bei Delir}

\begin{tabular}{|ll|}
\hline Untersuchung & diagnostischer Rückschluss auf \\
\hline Anamnese & Substanzentzug, Demenz \\
\hline Temperatur & Entzündung, Sepsis \\
\hline Blutbild & Anämie, Exsikose, Entzündung \\
\hline Elektrolyte, Osmolarität & $\begin{array}{l}\text { Exsikose, hyperosmolares Koma, Elektrolyt- } \\
\text { Entgleisung, Hypoparathyreodismus }\end{array}$ \\
\hline Glucose & Hypo-, Hyperglykämie \\
\hline Leberwerte & Leberversagen \\
\hline Retentionswerte & Nierenversagen \\
\hline Arterielle Blutgasanalyse & respiratorische Insuffizienz \\
\hline$T_{3}, T_{4}$, TSH & Hypo-, Hyperthyreose \\
\hline CRP & Entzündung \\
\hline Vitamin B 12 & Hypovitaminose \\
\hline Urinanalyse & Harnwegsinfekt, Substanzmissbrauch \\
\hline EKG & kardiale Ursachen \\
\hline Röntgen-Thorax & kardiale und pulmonale Ursachen \\
\hline EEG & Epilepsie \\
\hline CCT, MRT & primär zerebrale Ursachen \\
\hline
\end{tabular}

35\% der Patienten vorkommenden isolierten Halluzinosen und dopamininduzierten Psychosen zu unterscheiden, bei denen zumeist als nicht bedrohlich empfundene optische Halluzinationen ohne wahnhaftes Erleben bei klarem Bewusstsein auftreten. Halluzinationen beim deliranten Syndrom haben hingegen zumeist bedrohlichen Charakter und sind immer auch mit einer Bewusstseinsstörung verbunden.

\section{Kardiaka}

Bereits Duroziez beschrieb 1874 ein durch Digitalispräparate ausgelöstes Delir und fasste die Symptomatik unter dem Begriff des deliere digitalique zusammen (13). Die Thematik wurde in der Folgezeit ignoriert, bis Smith 1938 über das vermehrte Vorkommen von zentralnervösen Nebenwirkungen der Digitalistherapie bei älteren Patienten berichtete (58). Delirien können die erste und einzige Symptomatik einer Digoxinintoxikation sein (57). Delirante Symptomatik ist eine häufige Komplikation der Therapie mit Digoxin (30) und kann bereits unter therapeutischen Serumspiegeln auftreten $(14,23)$. In einer großen retrospektiven kanadischen Datenanalyse über gemeldete Medikamentennebenwirkungen fanden sich 264 Berichte über Digoxinnebenwirkungen, 213 Fälle davon waren Delirien (46).
Die meisten der Klasse-I-Antiarrhythmika haben anticholinerge Nebenwirkungen. Entsprechen liegen Berichte über delirante Syndrome mit psychotischer Symptomatik für Procainamid (49), Quinidin (11, 27), Lidocain (50) und Tocainid $(4,9)$ vor. Auch hier kann eine delirante Symptomatik bei Dosierungen im therapeutischen Bereich auftreten, nur bei Quinidin scheinen Delirien Folge eine Intoxikation zu sein. Für Amiodaron, ein Klasse-III-Antiarrhythmikum, liegen kasuistische Berichte über Delirien vor $(3,65)$.

\section{Kortikosteroide}

$\mathrm{Zu}$ den wichtigen unerwünschten Wirkungen einer Steroidtherapie gehören, neben den affektiven Störungen und der Induktion von akuten oder chronischen kognitiven Störungen, delirante Syndrome (48, 54). Nicht medikamentös bedingter Hyperkortisolismus führt zu Delirien und demenziellen Syndromen $(36,44)$. Iatrogen können letztere auch durch eine Langzeitbehandlung mit Kortikoiden bedingt sein, zur Ausbildung von deliranten Syndromen kommt es dagegen vor allem unter kurzzeitiger Hochdosistherapie $(47,61)$. Delirien wurden auch als Entzugssymptomatik nach langjähriger Kortikosteroidtherapie berichtet (8). In der Literatur sind eine Vielzahl von kortisoninduzier- 
ten Psychosen beschrieben (54), wobei die Rolle des Kortisols für die Genese psychotischer Syndrome unumstritten ist. Die genauen pathophysiologischen Mechanismen, die bei der Entstehung von unerwünschten psychotropen Nebenwirkungen mitwirken, sind allerdings noch unzureichend geklärt (48). Eine wichtige Rolle könnte eine Dysbalance gabaerger und monoaminerger Neurotransmittersysteme spielen. Darüber hinaus wurden eine genetische Vulnerabilität, die unterschiedliche enzymatische Eliminationsfähigkeit der Leber und die Exkretionsleistung der Niere sowie paranormale Blutplasmakonzentrationen von Medikamenten diskutiert $(32,60)$.

\section{Antibiotika und Chemo- therapeutika}

Es liegen unzählige Berichte über Delirien und psychotische Syndrome unter Therapie mit den unterschiedlichsten Antibiotika vor (59). In der Literatur werden als Risikofaktoren für die Ausbildung einer deliranten Symptomatik unter Antibiotikatherapie genannt $(43,53,59)$ :

- psychiatrische Vorerkrankung

- schwere somatische Erkrankung

- fortgeschrittenes Lebensalter

- eingeschränkte Nierenfunktion

- hohe Dosierung und/oder intravenöse/intrathekale Verabreichung

- erhöhte Permeabilität der BlutHirn-Schranke.

Eine genaue Einschätzung der neurotoxischen Wirksamkeit der verschiedenen Antibiotikaklassen ist nach der gegenwärtigen Datenlage schwierig, dennoch liegen die meisten Berichte zu Delirien für Penicilline, Cephalosporine und Quinolone vor $(1,12,43,53,55,64)$. Die Inhibition der GABAergen Neurotransmission wird als eine der möglichen Ursachen für die delirogene Wirkung der Fluoroquinolone und Penicilline angesehen $(1,29)$. Auch unter Fungiziden und Antimalariamitteln sind delirante Syndrome beschrieben (39).

Delirien können in Folge einer Chemotherapie oder einer Behandlung mit Zytokinen auftreten $(2,51$,
$66,67)$. Aufgrund der in der onkologischen Chemotherapie häufigen Polypharmazie lässt sich die verursachende Substanz nur schwierig identifizieren, und insbesondere bei ZNS-Tumoren oder Metastasen ist die Ätiologie deliranter Syndrome oft schwierig zu bestimmen. Insbesondere unter den Antibiotika, die zu den am häufigsten verschriebenen Medikamentengruppen gehören, wurden wiederholt psychische Störungen in Form von halluzinatorischen Syndromen oder deliranten Zustandsbildern beschrieben (15). Vor allem die Gyrasehemmer können unerwünschte ZNS-Effekte auslösen, was in früheren Zulassungs- und Postmarketingstudien mit ermittelten Häufigkeiten von 0,9 bis $2,2 \%$ dokumentiert wurde $(28,42,52,62)$. In der psychiatrischen Universitätsklinik München wurden im Rahmen des psychiatrischen Konsiliardienstes in den Jahren von 1990 bis 1994 alle Verdachtsfälle für psychopathologische Auffälligkeiten nach der Einnahme von Gyrasehemmern analysiert, wobei die retrospektive Auswertung von 4189 Berichten bei 29 Patienten den Verdacht auf das Vorliegen einer UAW mit psychiatrischer Symptomatik unter Gyrasehemmern ergab (24).

\section{Therapie}

Die Therapie der Wahl in der Behandlung medikamenteninduzierter Psychosen und deliranter Syndrome ist in erster Linie die Reduktion oder das Absetzen der potenziell psychotropen Substanz. In einzelnen Fällen kann auch eine Veränderung der Applikationsart zu einer Remission führen. Zumeist ist eine psychiatrisch-konsiliarische Betreuung und Behandlung hinreichend, bei schwerem Ausprägungsgrad der Symptomatik kann allerdings eine antipsychotische Therapie und gegebenenfalls eine stationär psychiatrische Behandlung nicht zu umgehen sein. Ist eine medikamentöse Therapie erforderlich, können antipsychotisch vor allem Haloperidol oder Risperidon eingesetzt werden, zur Sedierung die niedrigpotenten Neuroleptika Melperon oder Pipamperon. Bei Intoxikation mit anticho- linergen Substanzen, Neuroleptika auf Phenothiazinbasis, Antidepressiva, Antihistaminika oder Alkohol hat sich auch der Einsatz von Physostigmin bewährt. Insbesondere bei Parkinsonpatienten sollte allerdings eine neuroleptische Therapie mit Clozapin oder Quetiapin erfolgen, da andere Antipsychotika zu einer Verschlechterung der Parkinsonsymptomatik führen. Bei älteren Patienten sollte die Behandlung immer mit niedrigen Tagesdosen und langsamer Aufdosierung begonnen werden.

\section{Drug-induced psychosis}

The number of drugs that can induce psychiatric symptoms as side-effect is large. These drugs are often prescribed and in therapeutic use in other medical disciplines. Many of these drugs have side effects on the central nervous system and can induce psychotic symptoms. Frequently, these side effects are not diagnosed, not identified as drug side-effects or misdiagnosed as psychiatric disorders. The following article presents an overview of the important drugs that can induce psychosis and of the therapy of these psychotic syndromes.

\section{Key words}

delirium - drug induced - psychosis

\section{Literatur bei der Redaktion oder unter www.psychoneuro.info}

\author{
Korrespondenzadresse: \\ Dr. med. Christian Prüter \\ Gangelter Einrichtungen \\ Krankenhaus Maria Hilf \\ Fachkrankenhaus für Psychiatrie \\ und Psychotherapie \\ Bruchstraße 6 \\ 52538 Gangelt \\ chprueter@yahoo.de
}

